\title{
QUANTUM FLUIDS IN NANOPORES
}

\author{
NATHAN M. URBAN \\ Department of Physics, Pennsylvania State University \\ University Park, Pennsylvania 16802-6300, USA \\ nurban@phys.psu.edu \\ MILTON W. COLE \\ Department of Physics, Pennsylvania State University \\ University Park, Pennsylvania 16802-6300, USA \\ mwc@psu.edu
}

\begin{abstract}
We describe calculations of the properties of quantum fluids inside nanotubes of various sizes. Very small radius $(R)$ pores confine the gases to a line, so that a one-dimensional (1D) approximation is applicable; the low temperature behavior of $1 \mathrm{D}{ }^{4} \mathrm{He}$ is discussed. Somewhat larger pores permit the particles to move off axis, resulting eventually in a transition to a cylindrical shell phase - a thin film near the tube wall; we explored this behavior for $\mathrm{H}_{2}$. At even larger $R \sim 1 \mathrm{~nm}$, both the shell phase and an axial phase are present. Results showing strong binding of cylindrical liquids ${ }^{4} \mathrm{He}$ and ${ }^{3} \mathrm{He}$ are discussed.
\end{abstract}

\section{Introduction}

The discovery of carbon nanotubes has provided a playground for theoretical physics analogous to that $(\sim 1970)$ based on the discovery of adsorption on flat, wellcharacterized surfaces. In the former case, excitement arises from the tantalizing possibility that one-dimensional (1D) physics can be tested by studying adsorbed gases near nanotubes, just as studies of monolayer films provided tests of $2 \mathrm{D}$ physics.

Many groups have explored the properties of quantum fluids on the external surface of nanotube bundles and the interstitial regions within the bundles, stimulated by both the intriguing geometry and several experimental results!1234156178 Our group has predicted several novel phase transitions for interstitial quantum fluids, including a high temperature (liquid-vapor) condensation and a BEC that exhibits 4D (!) thermodynamic properties $[23]$ This paper summarizes instead diverse results concerning quantum fluids inside single nanotubes, obtained with a variety of methods. These studies are far from complete, with significant theoretical questions yet to be answered. The following section discusses the case of ${ }^{4} \mathrm{He}$ in $1 \mathrm{D}$, with applications to small radius $(R)$ pores. Section 3 explores the behavior of absorbed $\mathrm{H}_{2}$ as $R$ increases, so that the $1 \mathrm{D}$ approximation breaks down. Section 4 discusses the nature of films in large pores $(R \sim 1 \mathrm{~nm})$, where one encounters both a "cylindrical shell" phase of the film on the surface and a so-called "axial" phase, which is very much like the $1 \mathrm{D}$ system in the small $R$ case. 
Throughout this paper we omit the details of both the adsorption potential and the techniques used in the calculations. Those can be found in existing publications, as well as a thesis and longer article currently being drafted.4 Our emphases are new results, qualitative behavior, and significant open questions.

\section{Behavior in the 1D Limit}

The $1 \mathrm{D}{ }^{4} \mathrm{He}$ system is interesting for several reasons. One is that the liquid is barely bound (by about $1.7 \mathrm{mK}$ ) and very rarefied (mean spacing about $2.7 \mathrm{~nm}$ !); in fact, the venerable Lennard-Jones (LJ) pair potential is too weakly attractive to produce this bound state ${ }^{56}$ A related fact is that the threshold interaction strength for 1D binding of the liquid state coincides with that of the 1D dimer ${ }^{6}$ Recently, L.W. Bruch and C. Carraro (private communications) have shown that the cohesive energy of the 1D many-body system has the 1D dimer binding energy as a lower limit, the two energies possibly coinciding. There is a closely related, intriguing aspect to the dimer problem. Consider the three-dimensional (3D) dimer problem, focusing on the $s$-wave channel. The radial Schrödinger equation for that problem coincides with the Schrödinger equation for the 1D dimer. A key difference between $D=1$ and $D=3$ is the requirement that the wave function $\psi(r)$ vanish at $3 \mathrm{D}$ separation $r=0$. However, this difference is inconsequential for a $1 \mathrm{D}$ system involving hard-core interactions. Hence, the 3D wavefunctions and spectra coincide, at least for the $s$-channel, with those of the 1D problem. Putting all of this information together, it might be "expected" theoretically that all of the three energies agree, with the common value $1.7 \mathrm{mK}$.

Recently, we have studied the thermal properties of $1 \mathrm{D}$ liquid ${ }^{4} \mathrm{He}$, using the path integral method 1314 If one were to anticipate the behavior theoretically, one might treat the system with the Landau model, based on elementary excitations above the ground state. In the limit that the low-lying excitations are phonons, with $T=0$ speed $s(\rho)$ at density $\rho$, this model predicts that the energy per particle $\Delta E$ (relative to the ground state energy) satisfies

$$
\begin{aligned}
\Delta E(T) & =F\left(k_{B} T\right)^{2}, \\
F & =\frac{\pi / 6}{\hbar s \rho} .
\end{aligned}
$$

Preliminary results of the path integral calculations, in Fig. 1] are consistent with this prediction at low $T$ and high $\rho$; e.g., values of the coefficient $F$ in Eq. 11 fit to the data at $\rho=2.5 / \mathrm{nm}$ (and higher) agree with the value predicted by Eq. 2 At $\rho=$ $2 / \mathrm{nm}$ and below, instead, the values of $F$ begin to disagree and the departure from the quadratic dependence occurs at lower $T$. The latter is not surprising because the $T^{2}$ dependence of $\Delta E$ is expected only far below the 1D Debye temperature, $\Theta=\hbar s \rho /\left(k_{B} \pi\right)$. Since $\Theta \sim 8 \mathrm{~K}$ at $\rho=2 / \mathrm{nm}$ and $\Theta \sim 30 \mathrm{~K}$ at $\rho=2.5 / \mathrm{nm}$, different behavior of $\Delta E$ is expected for the two densities at $5 \mathrm{~K}$.

Departure from simple model behavior at very low $\rho$ is also expected on general grounds, since the speed of sound becomes imaginary below the spinodal density, 


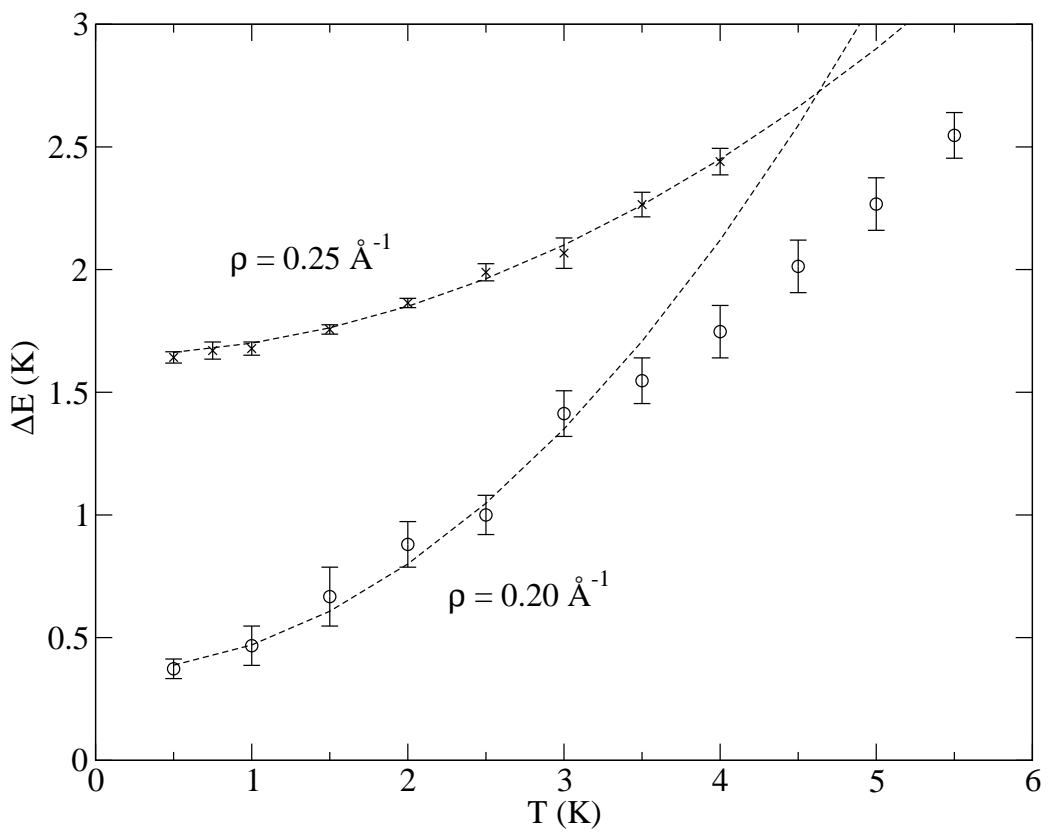

Fig. 1. Energy per molecule of 1D ${ }^{4} \mathrm{He}$ at $\rho=2.0$ and 2.5/nm. Quadratic curves are based on the Landau-like model. (From Ref. 4 )

$\rho \sim 0.245 / \mathrm{nm}$ (compared to an equilibrium density $0.36 / \mathrm{nm}$ ). The phonon theory has no meaning at such low $\rho$. The observed departure from Landau-like predictions occurs at much higher density than that, however. This empirical behavior remains to be understood. At very low density, one might be inclined to use a virial expansion. Šiber has evaluated the first virial correction to the $1 \mathrm{D}{ }^{4} \mathrm{He}$ system. His results show significant departure from the classical specific heat $(1 / 2$ Boltzmann per atom) at relatively high $T$, even for very low density. This has nothing to do with exchange, which does not contribute because of the hard core repulsion $\frac{9}{9}$ One expects higher order virial terms to contribute in addition, making this system particularly interesting to explore experimentally.

\section{Spreading Away From the Axis}

The $T=0$ properties of $1 \mathrm{D} \mathrm{H}_{2}$ were first calculated by Boronat, Gordillo, and Casulleras ${ }^{[7]}$ Here, we report preliminary results of finite $T$ behavior as the radius is increased from a very small value to $R=0.7 \mathrm{~nm}$.

Assuming a Lennard-Jones interaction between the gas adsorbate molecules and the inner pore surface, as adsorbate density is increased the gas will begin to adsorb onto the pore as a cylindrical shell film, at a distance from the surface on the order of the LJ parameter $\sigma$. However, we expect that if the radius $R$ of the pore is less than this characteristic distance, the gas-surface repulsion will heavily restrict the 
transverse motion of the gas, and the two-dimensional (2D) shell will collapse into a 1D line of molecules on the pore axis.

Geometrically, this axial compression at small pore radii exhibits itself in the nanopore potential as a transition from a minimum near $r \sim R-\sigma$ and a maximum at $r=0$ (i.e., an off-axis potential well) when $R \gtrsim \sigma$, to a simple minimum at $r=0$ when $R \lesssim \sigma$ (an on-axis potential well). For the case of an infinitesimally thin tube, this transition is analytically known to occur at $R \simeq 1.212 \sigma ! \sqrt{10}$ We do not know an analogous analytic result for the case studied here, a pore within bulk material, but numerically the transition point is similar.

For concreteness, we chose to study pores in $\mathrm{MgO}$ glass. The $\mathrm{H}_{2}$ - $\mathrm{MgO}$ LJ interaction parameters are given by $\sigma=2.014 \AA, \epsilon=45.91 \mathrm{~K}$.11 The $\mathrm{H}_{2}-\mathrm{H}_{2}$ interaction potential was taken to be of the Silvera-Goldman form.12 The system was studied numerically via the path integral Monte Carlo algorithm.1314 Temperatures between $T=0.5 \mathrm{~K}$ and $3 \mathrm{~K}$, radii between $R=2$ and $7 \AA$, and densities of $\rho=\left(2.55 \times 10^{-3} / \AA\right) / R^{2}$ and $\rho=\left(7.13 \times 10^{-2} / \AA\right) / R^{2}$. (The densities correspond to choosing a fixed number $N=1$ or $N=28$ of particles in a simulation cell of radius $R$ and fixed length $125 \AA$, with periodic longitudinal boundary conditions.)

The results obtained were very similar for both densities and all ranges of temperature and radii studied. A representative plot of the $\mathrm{H}_{2}$ radial density distribution and the pore potential is given in Fig. 2 for radii between $R=2.5$ and 3.25 $\AA$.

The lowest pore size depicted, $R=2.5 \AA$, is just beyond the transition point (2.45 $\AA$ for the tubular case) to an off-axis minimum, where the $\mathrm{H}_{2}$ should switch from a $1 \mathrm{D}$ axial phase to a $2 \mathrm{D}$ cylindrical shell. This is reflected in the potential energy curve (Fig. 2), which is very shallow for $R=2.5 \AA$. Correspondingly, we see that at this radius the $\mathrm{H}_{2}$ molecules are still concentrated on the axis. However, with a remarkably small increase in pore radius, by $0.25-0.5 \AA$, the distribution rapidly shifts to peak off axis, signifying the onset of the shell phase. It is interesting that the transition from axial to shell phases coincides in $R$ with the shift of the classical potential minimum from on- to off-axis, even in the presence of quantum effects and interparticle interactions. One expects larger effects of interactions at higher density.

It should be noted that the radii depicted in Fig. 2 are unphysically small for $\mathrm{MgO}$ pores of realistic size. We chose to study them despite this problem in order to theoretically study an axial to cylindrical shell transition in a pore geometry, which can only occur for small pores which are highly confining. In addition, there are some systems of larger radii in which the first layer to be adsorbed becomes a rigidly bound film, confining a fluid phase to a very localized vicinity of the pore axis 15 Such possibilities help to justify the study of quasi-1D fluids.

\section{Large Pore Phenomena}

Relatively few simulation studies have been carried out for quantum fluids in "typical" size nanotubes, $R \sim 0.6$ to $1 \mathrm{~nm}$. Path integral calculations of Gatica et al $\frac{16}{16}$ 

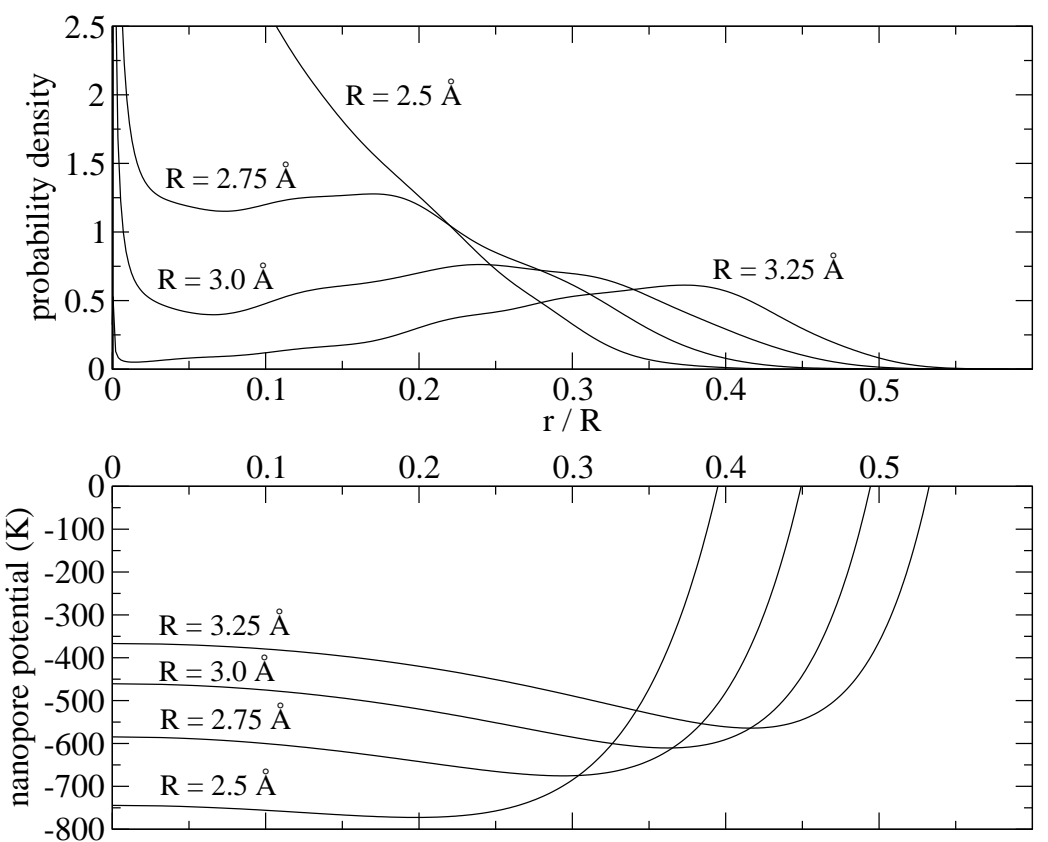

Fig. 2. $\mathrm{H}_{2}$ radial probability density distribution (at $T=0.5 \mathrm{~K}, \rho=\left(2.55 \times 10^{-3} / \AA\right) / R^{2}$ ) and $\mathrm{MgO}$ pore potential, for pore radii $R=2.5,2.75,3.0$, and $3.25 \AA$, as functions of dimensionless radius $r / R$. (Radial densities near $r=0$ are exaggerated due to finite size effects after normalizing the radial distribution by $1 /(2 \pi r)$ to obtain the probability density.)

reported the behavior of $\mathrm{H}_{2}$ over a limited range of $R$ at $T=10 \mathrm{~K}$. One of the more interesting phenomena is the pore-filling transition, shown for $\mathrm{H}_{2}$ in Fig. [3] 16 low $\mu$, all of the molecules are localized within a thin layer, at $r=0.3 \mathrm{~nm}$, located near the distance of closest approach to the nanotube. Above a threshold value of $\mu$, the axial phase appears and grows rapidly with increasing $\mu$. This axial phase can be thought of as an independent 1D phase. A close analogy is the behavior of the second layer film of $\mathrm{He}$ or $\mathrm{H}_{2}$ on the surface of graphite, often treated by assuming that the only role of the first layer is to provide a holding potential 17

Analytical and numerical problems associated with matter in cylindrical geometry are often computationally demanding, motivating the use of simplifying models that (we hope) capture the essential physics. Recently, we have explored such a description of the shell phase; the model assumes that all particles are constrained to lie on a cylindrical surface, $r=R$. One might expect that by varying $R$ between $R=0$ and $R=\infty$, one interpolates smoothly between $1 \mathrm{D}$ and $2 \mathrm{D}$ behaviors. This is naïve; instead, an intriguing "anomaly" arises: a significant enhancement of the binding occurs when the diameter of the cylinder, $d=2 R$, is comparable to the equilibrium separation $r_{\text {min }}$ in the pair potential. The condition $d \sim r_{\text {min }}$ corresponds (for LJ interactions) to $\sigma / R \sim 1.7$. Indeed, this argument does explain the $R$ value corresponding to the maximum cohesive energy (seen in Fig. (4) of the "cylindrical 


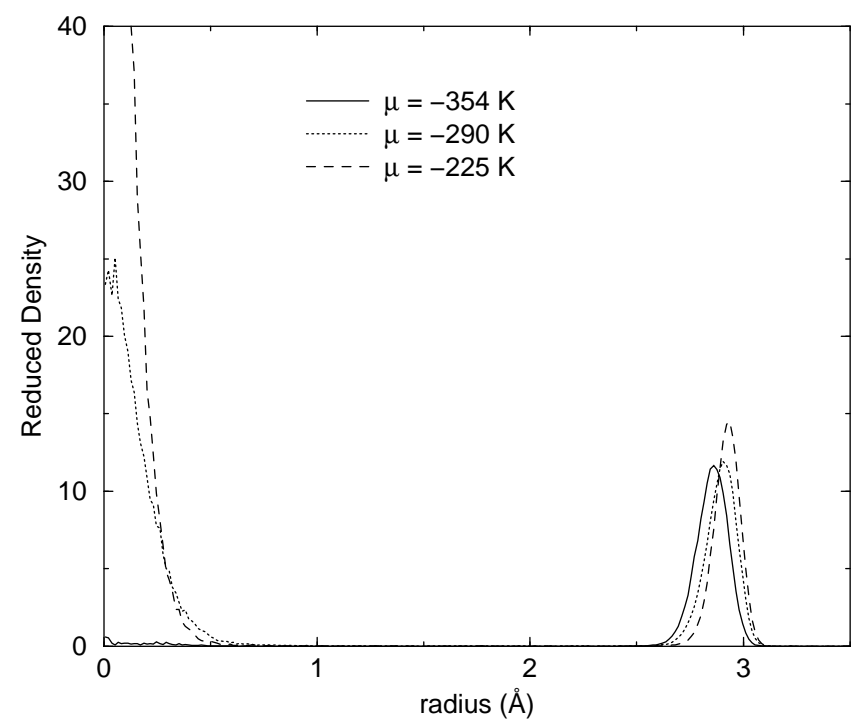

Fig. 3. Pore-filling transition of $\mathrm{H}_{2}$ in a tube of radius $0.6 \mathrm{~nm}$, from Gatica et al ${ }^{16}$ Results are densities as a function of $r$ at $10 \mathrm{~K}$ and indicated chemical potentials.

liquids" ${ }^{4} \mathrm{He}$ and ${ }^{3} \mathrm{He}$. The ${ }^{3} \mathrm{He}$ case is perhaps the most dramatic, because its liquid state does not exist in either $1 \mathrm{D}$ or $2 \mathrm{D}$, while the cylindrical liquid ${ }^{3} \mathrm{He}$ is found to have cohesive energy as high as $1.26 \mathrm{~K}$ for $R=0.18 \mathrm{~nm}$.

These are variational results, obtained with Jastrow and Slater-Jastrow wave functions for ${ }^{4} \mathrm{He}$ and ${ }^{3} \mathrm{He}$, respectively. Qualitatively similar, enhanced binding behavior was found for related problems involving similar binding problems on a cylindrical surface: $\mathrm{He}$ or $\mathrm{H}_{2}$ dimers, a crystalline lattice confined to a cylinder, and the virial coefficient of a classical fluid 18 The origin of this general behavior is that two interacting particles can maximally exploit a divergent "specific area" when the interatomic separation is favorable. This occurs when the particles are on opposite sides of the cylinder, with separation $\left|\mathbf{r}_{2}-\mathbf{r}_{1}\right|=d=r_{\text {min }}$. The specific area is defined as the cylindrical area residing within a separation interval $[r, r+d r]$, divided by $d r$. For completeness, we note that analogous results for the dimer binding have been found by Aichinger et al., using both the simple model of confinement on a cylinder and more realistic study of dimers inside a nanotube ${ }^{[19}$ The optimal binding value found for $R$ is very different in the two cases.

\section{Acknowledgments}

We are grateful to our collaborators (Massimo Boninsegni, Louis Bruch, Mercedes Calbi, Carlo Carraro, Silvina Gatica, and Milen Kostov) for many contributions to this project and to NSF for its support of this research. 


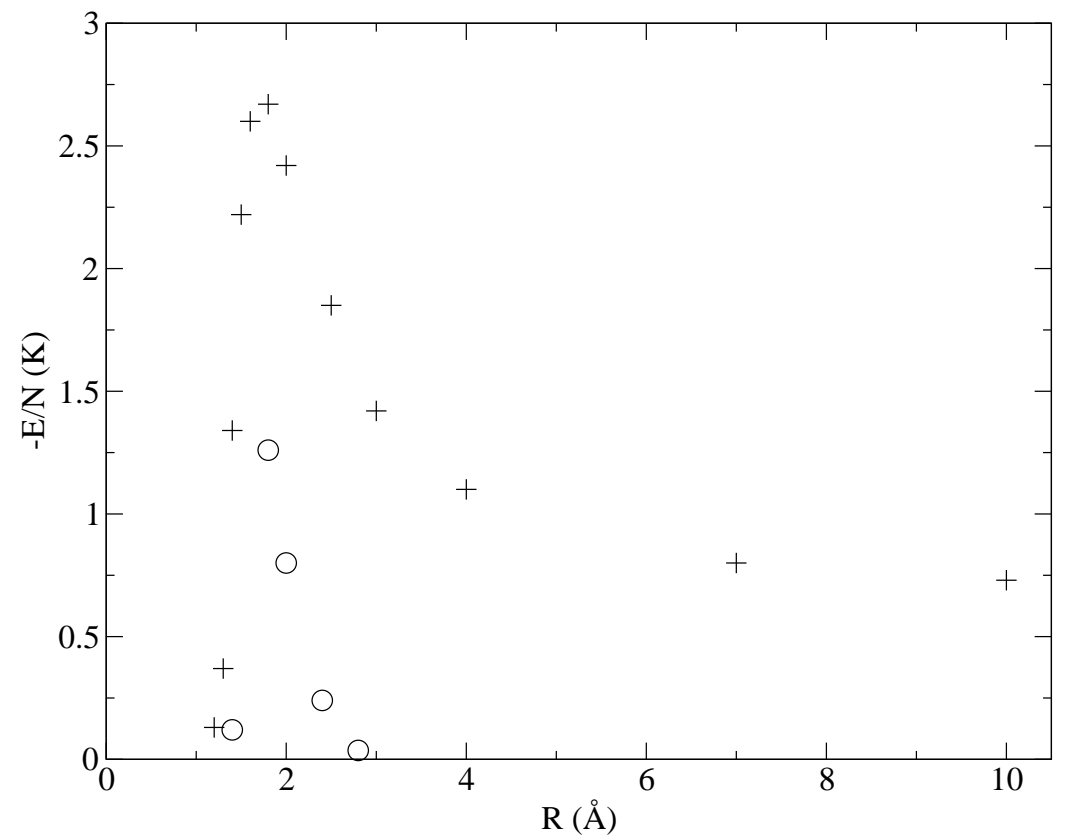

Fig. 4. Cohesive energy per atom of cylindrical liquids, as a function of $R$. Pluses are ${ }^{4}$ He data (from Kostov et al ${ }^{18}$ ) and circles are ${ }^{3} \mathrm{He}$ data (from C. Carraro, unpublished).

\section{References}

1. S. Ramachandran, T.A. Wilson, D. Vandervelde, D.K. Holmes, and O.E. Vilches, J. Low Temp. Phys. 134, 115 (2004); K.A. Williams, P.C. Eklund, M.K. Kostov, and M.W. Cole, Phys. Rev. Lett. 88, 165502 (2002); J.C. Lasjaunias, K. Biljakovic, J.L. Sauvajol, and P. Monceau, Phys. Rev. Lett. 91, 025901 (2003); J.V. Pearce, M.A. Adams, O.E. Vilches, M.R. Johnson, and H.R. Glyde, Phys. Rev. Lett. 95, $185302(2005)$

2. M.M. Calbi, F. Toigo, and M.W. Cole, Phys. Rev. Lett. 86, 5062 (2001); C. Carraro, Phys. Rev. Lett. 89, 115702 (2002).

3. F. Ancilotto, M.M. Calbi, S.M. Gatica, and M.W. Cole, Phys. Rev. B 70, 165422 (2004).

4. N.M. Urban, M. Boninsegni, and M.W. Cole, unpublished.

5. M. Boninsegni and S. Moroni, J. Low Temp. Phys. 118, 1 (2000); J. Boronat, M.C. Gordillo and J. Casulleras, ibid. 126, 199 (2002).

6. E. Krotscheck, M.D. Miller, and J. Wojdylo, Phys. Rev. B 60, 13028 (1999); E. Krotscheck and M.D. Miller, Phys. Rev. B 60, 13038 (1999).

7. M.C. Gordillo, J. Boronat, and J. Casulleras, Phys. Rev. Lett. 85, 2348 (2000).

8. M. Boninsegni, S. Lee and V.H. Crespi, Phys. Rev. Lett. 86, 3360 (2002).

9. L.W. Bruch, Phys. Rev. B 70, 016501 (2004); A. Šiber, Phys. Rev. B 70, 016502 (2004).

10. G. Stan and M.W. Cole, Surf. Sci. 395, 280 (1998).

11. G. Vidali, G. Ihm, H.Y. Kim, and M.W. Cole, Surf. Sci. Rep. 12, 133 (1991).

12. I.F. Silvera and V.V. Goldman, J. Chem. Phys. 69, 4209 (1978).

13. D.M. Ceperley, Rev. Mod. Phys. 67, 279 (1995). 
14. M. Boninsegni, J. Low Temp. Phys. 141, 27 (2005).

15. J. Taniguchi, A. Yamaguchi, H. Ishimoto, H. kegami, T. Matsushita, N. Wada, S.M. Gatica, M.W. Cole, F. Ancilotto, S. Inagaki, and Y. Fukushima, Phys. Rev. Lett. 94, 065301 (2005).

16. S.M. Gatica, G. Stan, M.M. Calbi, J.K. Johnson, and M.W. Cole, J. Low Temp. Phys. 120, 337 (2000).

17. M. Pierce and E. Manousakis, Phys. Rev. Lett. 81, 156 (1998).

18. M.K. Kostov, M.W. Cole, G.D. Mahan, C. Carraro and M.L. Glasser, Phys. Rev. B 67, 075403 (2003); M.M. Kostov, M.W. Cole, and G.D. Mahan, Phys. Rev. B 66, 075407 (2002); M.M. Calbi, S.M. Gatica, M.J. Bojan, and M.W. Cole, Phys. Rev. E 66, 061107 (2002).

19. M. Aichinger, S. Kilic, E. Krotscheck, and L. Vranje, Phys. Rev. B 70, 155412 (2004); S. Kilic, E. Krotscheck, and R. Zillich, J. Low Temp. Phys. 116, 245 (1999). 